specifically targets the CNS. Furthermore, the authors established that intravenous antiviral siRNA treatment could protect mice infected with fatal viral encephalitis: survival in a treatment group was around $80 \%$, whereas all control animals died.

Transvascular delivery of siRNA to the CNS mediated by RVG-9R results in a gene-silencing effect comparable to that of direct injection, with the effect declining at a similar rate. This novel approach could provide a safe and noninvasive means of treating neurological disease with siRNA, and further studies are indicated.

Original article Kumar P et al. (2007) Transvascular delivery of small interfering RNA to the central nervous system. Nature 448: $39-43$

\section{Inflammatory MS lesions linked to characteristic metabolic profile in cerebrospinal fluid}

An understanding of the metabolic alterations underlying the different manifestations of multiple sclerosis (MS) might facilitate the development of tailored treatment regimens. Several studies have examined the metabolic profiles of cerebrospinal fluid (CSF) from patients with MS, but the results have been conflicting. Lutz and co-workers hypothesized that these inconsistent results might stem from variations in the MS populations studied, given the numerous stages and manifestations of this disease. In a highly homogeneous MS population solely comprising untreated patients presenting with clinically isolated syndrome (CIS), Lutz et al. examined the association between MS inflammatory activity and CSF metabolic profiles.

CSF was obtained by lumbar puncture from 10 controls and from 21 patients with and 12 patients without inflammatory plaques. Proton magnetic resonance spectroscopy was used to quantify 27 metabolites. In comparison with controls, both CIS groups showed significant increases in levels of fructose $(P<0.05)$, but not in those of other metabolites. When the metabolite profiles for the two CIS groups were compared, the researchers noted markedly higher levels of $\beta$-hydroxyisobutyrate and lactate in the CIS group with active plaques than in the CIS group without plaques. Spearman rank analysis demonstrated a significant correlation between lactate levels and the number of inflammatory plaques $(P=0.002)$. These results suggest a close association between inflammatory plaque activity and organic acid metabolism.

Original article Lutz NW et al. (2007) Inflammatory multiple-sclerosis plaques generate characteristic metabolic profiles in cerebrospinal fluid. PLoS ONE 2: e595

\section{Glioblastoma multiforme proximity to neural stem cells predicts malignancy}

Glioblastoma multiforme (GBM) is a heterogeneous disease, and various progenitor cells have been implicated in the origin of GBM tumors. Tumors with the greatest migratory potential are suspected to arise from neural stem cells. As part of a brain tumor imaging research study, Lim et al. examined the link between the severity of GBM and the tumor's spatial relationship to brain regions associated with neural stem cells, in particular the subventricular zone (SVZ).

The lesions of 53 consecutive patients newly diagnosed with GBM were characterized by $\mathrm{MRI}$ before and after surgery. The investigators found that tumor proximity to the SVZ and cortical involvement were significant predictors of a multifocal phenotype, and they categorized tumors into four groups of descending malignancy according to these criteria. Interestingly, tumor volume was not significantly associated with tumor severity. Tumors that were in contact with the SVZ and that infiltrated the cortex (Group I) were most likely to be multifocal $(P=0.001)$, and almost all recurrences of Group I tumors were invasive and distal to the original tumor site. By contrast, tumors in Group IV (neither contacting the SVZ nor infiltrating the cortex) were never multifocal, and recurrences were always contiguous with the initial lesions. The authors hypothesize that Group I tumors are derived from SVZ cells, and are more invasive than other tumor types as a consequence.

Predicting the recurrence and migratory potential of tumors would have important implications for treatment decisions. Further studies are needed to determine the clinical utility of classifying GBM lesions by MRI.

Original article Lim DA et al. (2007) Relationship of glioblastoma multiforme to neural stem cell regions predicts invasive and multifocal tumor phenotype. Neuro Oncol [doi: 10.1215/15228517-2007-023] 